November 13, 2018 23:41 MANUSCRIPT OrbifoldBreaking

Modern Physics Letters A

(C) World Scientific Publishing Company

\title{
GAUGE SYMMETRY BREAKING IN ORBIFOLD MODEL BUILDING
}

\author{
MICHELE TRAPLETTI \\ Institut für Theoretische Physik, Ruprecht-Karls-Universität Heidelberg, \\ Philosophenweg 16, D-69120, Heidelberg Germany. \\ m.trapletti@thphys.uni-heidelberg.de
}

\begin{abstract}
We review the gauge symmetry breaking mechanism due to orbifold projections in orbifold model building. We explicitly show the existence of a scale of breaking if such a symmetry breaking is due to freely-acting orbifold operators only, i.e. in case the breaking is realized non-locally in the internal space. We show that such a scale is related to the compactification moduli only, and that there are no extra continuous parameters, at least in semirealistic models with $\mathcal{N}=1$ SUSY in four dimensions. In this sense, the mechanism is peculiarly different from the standard Higgs (or Hosotani) symmetry breaking mechanism. We show that the mechanism also differs from that present in standard orbifold models where, even in presence of discrete Wilson lines, a scale of breaking is generically missing, since the breaking is localized in specific points in the internal space.

We review a set of background geometries where the described non-local breaking is realized, both in the case of two and six extra dimensions. In the latter case, relevant in string model building, we consider both heterotic and open string compactifications.
\end{abstract}

Keywords: string model; orbifold; grand unified theory.

PACS Nos.: 11.10.Kk, 11.25.Mj, 11.25.Wx, 12.10.-g

Preprint No: HD-THEP-06-27

\section{Introduction and outline}

Unification of Electroweak and Strong interaction couplings 1 , at a precise energy scale $^{2}$, is the main information we have about physics at energies much larger than $\mathrm{TeV}$. Given how elementary particles fit multiplets of a unified gauge group, it is natural to conclude that such unification is not an accident, but a crucial feature of Nature, that should be explained, rather then merely reproduced, by an extension of the Standard Model of Particle Physics.

Many attempts have been devoted to the construction of unified models, in a field theory 3 scenario, even in presence of one ${ }^{415}$ or two extra dimensions, as well as in string model building (for recent realistic constructions see ${ }^{7}$ ). In the latter, large gauge groups naturally arise, and a unified description of gauge and gravity interactions is also possible. In all the models a key feature is the gauge symmetry breaking mechanism, and in this paper we focus on such an issue: Our 
main aim is the study of the mechanism due to the presence of orbifold projections in (toroidal) orbifold models. We avoid a complete discussion of other symmetry breaking mechanisms, such as the one realized by continuous Wilson lines or by Higgs-like effects, as well as the kind of gauge symmetry breaking due to magnetic fluxes for the gauge fields or, in a dual picture, the one due to the presence of angles between D-branes in open string model building.

We distinguish between two different options, characterized by the details of the orbifold projection. Namely, it may act in the internal space as a rotation (with fixed points) or as a rototranslation (without fixed points i.e. freely).

In the rotational case the breaking is localized in the fixed points and, generically, there is no scale of breaking: the coupling constants of the unbroken gauge factors (namely, $S U(3), S U(2)$ and $U(1))$ run in different ways at all the energies. This implies that the unification of the couplings can be at best numerically reproduced, but not explained.

In the rototranslational case the breaking is non-local 8 . This implies that the couplings run in the same unified way between the high energy cutoff of the model (string scale) and an intermediate scale, related to the compactification scales. Then, from this scale down to low energy, the three couplings run in different ways, and so they split apart from each other $\underline{13}$. It is very natural to define such an intermediate scale as the symmetry breaking scale of the model.

The presence of such a scale is crucial in heterotic string model building, where the string scale is typically larger than the "observed" unification scale $M_{G U T}=3 \times$ $10^{16} \mathrm{GeV}$ 14: the hierarchy $M_{\text {string }} / M_{G U T}$ can be introduced via a compactification scale smaller then $M_{\text {string }}$, rather then by appealing to large threshold corrections at the string scale $15 \mathrm{~b}$.

We mainly focus on non-local breaking, describing its details in nontrivial compactifications. Since an orbifold model with a single orbifold operator/projection is an over-simplified system , we extend the analysis to orbifold models with enlarged orbifold group. In this case the gauge symmetry breaking is non-local only provided that all the gauge-symmetry-breaking orbifold operators have a free action 17 . Such a requirement imposes severe constraints on the allowed orbifolds/geometries; we

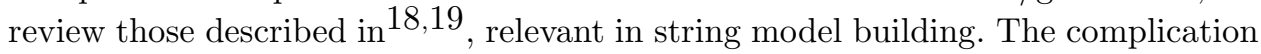
of having many orbifold operators has a relevant extra consequence. Namely, it ensures the absence of continues Wilson lines mimicking the kind of gauge symmetry breaking we are interested in, i.e. it ensures that the symmetry breaking scale is completely fixed by the compactification moduli. The mechanism is then peculiarly different from the Hosotani mechanism, where an extra continuous parameter, other

\footnotetext{
${ }^{a}$ Notice that such a construction is also a realization of the well known Scherk-Schwarz (SUSY) breaking mechanism 9 , as shown, in a string context, in 10 11]. (For similar constructions in a field theory context, see alsd 12 .)

${ }^{\mathrm{b}}$ For more recent alternatives addressing the $M_{\text {string }} / M_{G U T}$ hierarchy see alsd 16 .

${ }^{\mathrm{c}}$ Indeed, a toroidal orbifold with a single orbifold operator, acting freely, is typically inconsistent with the requirement of having a four-dimensional chiral spectrum.
} 
than the compactification scale, can be tuned to fix the breaking scale, exactly as in the standard Higgs mechanism. In this sense, the mechanism combines the absence of extra continuous parameters, typical of discrete Wilson lines breaking, with the presence of a breaking scale, typical of Hosotani/Higgs mechanism.

The Outline of the paper is the following

In Sect. 2 we consider field theory examples, in presence of two extra dimensions compactified on orbifolds. We first study two simplified models, one with rotational action, the other with rototranslational action, computing the running of the coupling constants. We show that only in the rototranslational case it is possible to have unification of the couplings at the compactification scale and unified running of the couplings between this scale and the cutoff. We extend the study to a third model with non-local breaking, discussed in $\frac{17}{17}$ and relevant also in 18 . We compute the symmetry breaking scale, finding its exact relation with the surface of the twodimensional space. We also show the absence of continuous Wilson lines mimicking the non-local gauge symmetry breaking. Finally, we extend the results to generic orbifolds, and comment about the gauge symmetry breaking due to discrete Wilson lines. We show that in the latter case a scale of breaking is present only in very specific constructions and is absent in the general case.

In Sect. 3 we study $6 \mathrm{~d}$ orbifold geometries, relevant in string model building since Type I and heterotic string compactifications on such backgrounds produce $4 \mathrm{~d} \mathcal{N}=$ 1 SUSY models with non-local gauge symmetry breaking. We comment about the details of open string model building 19 . We remind that, in presence of stacks of Dpbranes with $p<9$, the orbifold action can be external to the stacks, i.e. it can identify different stacks of D-branes. In this case the symmetry breaking reduces the rank of the gauge group. We comment about a purely external orbifold action, irrelevant for our purposes, a purely internal action, producing models with features close to the heterotic ones, and finally we address the issue of a mixed internal and external action. We show that in this case the combination of a rank-reducing orbifold with a discrete Wilson line can produce a model with gauge coupling unification at a scale fixed by the compactification scale.

\section{Acknowledgments}

It is a pleasure to thank Arthur Hebecker and Stefan Groot Nibbelink for discussions and comments on the draft.

\section{Gauge symmetry breaking via orbifold projections}

Our starting point is $S U(5)$ gauge theory defined on a $4+2$ dimensional spacetime. The two extra dimensions are compactified on a toroidal orbifold. Such a space is a $2 \mathrm{~d}$ torus with complex parameter $z=z_{1}+i z_{2}$ having periodicities $z_{i} \sim$ $z_{i}+2 \pi R_{i}$, in which we identify points that are symmetric under the action of the orbifold group $\mathcal{G}: z \equiv g(z) \forall g \in \mathcal{G}$. We consider bulk fields periodic in the internal dimensions, allowing non-trivial orbifold identifications, i.e. we consider a KaluzaKlein expansion reduced to the states that are invariant under the orbifold action. 

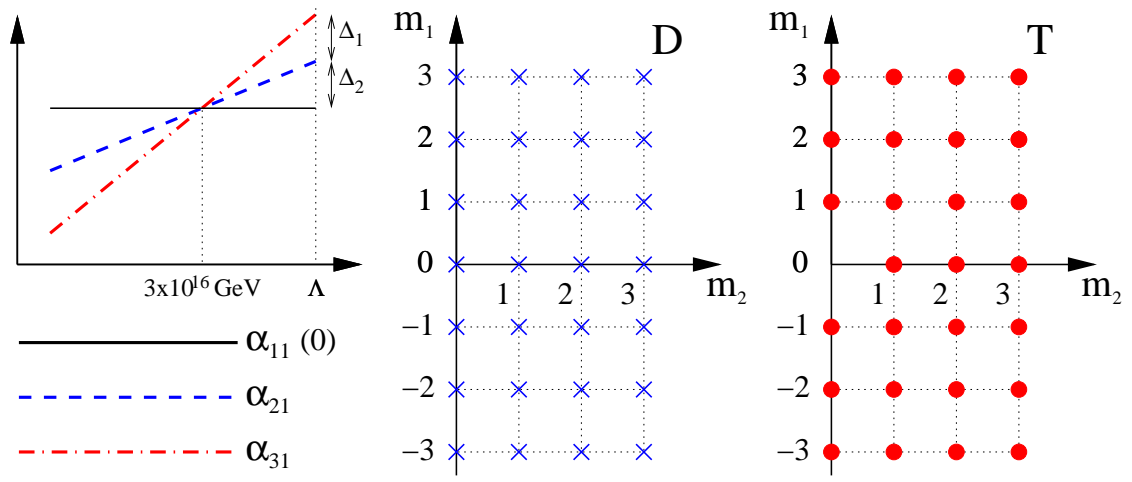

Fig. 1. Kaluza-Klein expansion of a fundamental representation of $S U(5)$ in the $T^{2} / Z_{2}$ orbifold. The figure in the middle shows the KK tower of the doublet (D), a blue cross in a point with coordinates $m_{1}, m_{2}$ indicates the presence in the tower of a single state with $K K$ mass $m^{2}=$ $m_{1}^{2} / R_{1}^{2}+m_{2}^{2} / R_{2}^{2}$. The figure on the right side shows the tower for the triplet $(T)$. A similar expansion holds for any SU(5) multiplets, this implies that the running of the difference of the inverse coupling constants is as given in the figure on the left side, i.e. it never stops up to the cutoff energy $\Lambda$. Thus, it can be consistent with low energy data either in case $\Lambda \sim 10^{16} \mathrm{GeV}$ or in the presence of threshold corrections $\Delta_{1}$ and $\Delta_{2}$ at the cutoff energy. In both cases the unification of the couplings at $3 \times 10^{16} \mathrm{GeV}$ is nothing else than a numerical accident.

As an example, consider a scalar field in the fundamental representation of $S U(5), \Phi^{a}\left(x^{\mu}, z\right)$. It has Kaluza-Klein (KK) expansion

$$
\Phi^{a}\left(x^{\mu}, z\right)=\sum_{m_{i}=-\infty}^{\infty} \Phi_{m_{1}, m_{2}}^{a}\left(x^{\mu}\right) e^{\frac{i m_{1} z_{1}}{R_{1}}+\frac{i m_{2} z_{2}}{R_{2}}} .
$$

The action of the orbifold operator $g$ is

$$
g: \Phi^{a}\left(x^{\mu}, z\right)=\gamma_{g}^{a b} \Phi^{b}\left(x^{\mu}, g(z)\right),
$$

with $\gamma_{g}$ the embedding of the orbifold group in the gauge bundle. We project out of the spectrum all the KK states that are not invariant under the action of $g$. Considering the $Z_{2}$ case $g(z)=-z$, we deduce the action of $g$ on the KK modes, and see that only the combinations

$$
\Phi_{m_{1}, m_{2}}^{a}+\gamma_{g}^{a b} \Phi_{-m_{1},-m_{2}}^{b}
$$

are left in the spectrum. We also deduce that, depending on $\gamma_{g}$, the orbifold identification breaks the gauge symmetry $\frac{45}{4}$, e.g. $S U(5) \rightarrow S U(3) \times S U(2) \times U(1)$.

In case an orbifold operator $g$ has fixed points, as in the $Z_{2}$ example given above, these result in singularities in the orbifold space itself, where extra states can be localized. These states arise, in a string model, due to consistency conditions (e.g. modular invariance in heterotic string model building), and must respect the gauge symmetry that is left unbroken in the singularities, i.e. the gauge symmetry preserved by the operator $g$.

We can now study the details of the gauge symmetry breaking, in particular the existence of an energy scale. To inspect it we compute the differential running $y^{5}$ 
of the $S U(3), S U(2)$ and $U(1)$ couplings, i.e. the running of the difference of the inverse couplings: $\alpha_{i j}=\alpha_{i}^{-1}-\alpha_{j}^{-1}$. Such a quantity can be written, in presence of two extra dimensions, as

$$
\alpha_{i j}\left(M_{Z}\right)=b_{i j ; 00} \log \left(\frac{\Lambda}{M_{Z}}\right)+\sum_{\left(m_{1} m_{2}\right) \neq(00)} b_{i j ; m_{1} m_{2}} \log \left(\frac{\Lambda}{\sqrt{m_{1}^{2} R_{1}^{-2}+m_{2}^{2} R_{2}^{-2}}}\right) .
$$

In Eq. (4) $M_{Z}$ is a generic energy scale, lower than any compactification scale, while $\Lambda$ is the high energy cutoff of the model; the coefficients $b_{i j ; m n}$ are defined as $b_{i j ; m n}=b_{i ; m n}-b_{j ; m n}$, with $b_{i ; m n}$ the standard $\beta$-function coefficients of the coupling $\alpha_{i}$, due to KK modes with mass $M^{2}=m_{1}^{2} / R_{1}^{2}+m_{2}^{2} / R_{2}^{2}$.

If there exists an energy scale $M_{G U T}<\Lambda$, such that for energies larger than $M_{G U T}$ the contribution of massless modes to $\alpha_{i j}$ is precisely canceled by that of massive modes, than Eq. (4) can be written as

$$
\alpha_{i j}\left(M_{Z}\right)=b_{i j ; 00} \log \left(\frac{M_{G U T}}{M_{Z}}\right)
$$

and we identify $M_{G U T}$ with the unification scale. Indeed, from Eq. (5) we deduce that the couplings run in a unified way from $\Lambda$ to $M_{G U T}$, and they split (unify) precisely at $M_{G U T}$. If such an $M_{G U T}$ is not present, then the gauge symmetry breaking occurs in the absence of a breaking scale.

\subsection{Rotational orbifold: $a T^{2} / Z_{2}$ model with localized gauge symmetry breaking}

As an example of rotational orbifold, we consider $S U(5)$ gauge theory on $T^{2} / Z_{2}$. We take $Z_{2}=\{I, g\}, g: z \rightarrow-z$. Given the generators of $S U(5)$ as $5 \times 5$ matrices, and choosing $\gamma_{g}$ as the diagonal matrix $\gamma_{g}=\operatorname{Diag}(-1,-1,-1,1,1)$, we have the desired gauge symmetry breaking $S U(5) \rightarrow S U(3) \times S U(2) \times U(1) 4$.

In order to study the differential running, we need the Kaluza-Klein expansion of the bulk fields. Consider, as an example, a scalar in the fundamental of $S U(5)$. It has KK expansion given in Eq. (2), with invariant states given by Eq. (3). In detail, for each $a=1, \ldots, 5$, there is a tower of states with $4 \mathrm{~d}$ mass $M^{2}=m_{1}^{2} / R_{1}^{2}+m_{2}^{2} / R_{2}^{2}$. The $g$-projection reduces these towers: for $a=4,5$ (i.e. for the fields forming a doublet of the unbroken $S U(2))$ there is a tower of states with $m_{2} \geq 0, m_{1} \in$ $\mathbb{Z}$. For $a=1,2,3$ (i.e. for the fields forming a triplet of the unbroken $S U(3)$ ), there is the same tower as for the doublet, but with missing massless mode $\left(m_{1}=\right.$ $0, m_{2}=0$ ), as shown in Fig. 1 At each massive KK level there is a single surviving doublet and a single surviving triplet, that combine and form a complete multiplet of $S U(5)$. Such a statement holds in the expansion of any multiplet of $S U(5)$ : it splits into submultiplets having identical massive KK tower, so that at any non-zero KK level they recombine in a full $S U(5)$ multiplet. In this way the contribution to the differential running due to massive KK modes is just zero, since it is the contribution 

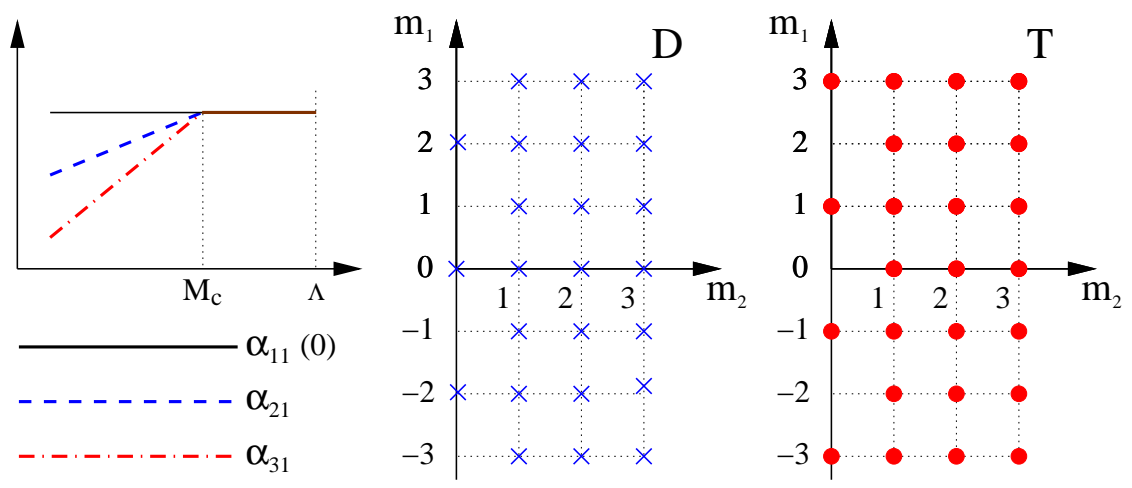

Fig. 2. Kaluza-Klein expansion of a fundamental representation of $S U(5)$ in the $T^{2} / Z_{2}^{\prime}$ orbifold. The figure in the middle shows the KK tower of the doublet (D), a blue cross in a point with coordinates $m_{1}, m_{2}$ indicates the presence in the tower of a single state with $K K$ mass $m^{2}=$ $m_{1}^{2} / R_{1}^{2}+m_{2}^{2} / R_{2}^{2}$. The figure on the right shows the tower for the triplet $(T)$. A similar expansion holds for any SU(5) multiplets, this implies that the running of the difference of the inverse coupling constants is as given in the figure on the left, i.e. it precisely stops, due to the KK contributions, at the compactification scale $M_{C}=1 / R_{1}$. In the model the breaking scale is then reproduced and explained provided $M_{C}=M_{G U T}=3 \times 10^{16} \mathrm{GeV}$.

of full multiplets of $S U(5)$ only, and, in Eq. (4),$b_{i j ; m_{1} m_{2}}=0$ for each $i, j$, provided that $\left(m_{1}, m_{2}\right) \neq(0,0)$.

Since the operator $g$ has fixed points, the model contains extra localized matter, that propagates in four dimensions only. This implies that its contribution to the differential running affects $b_{i j}$; 00 only. Thus, we conclude that

$$
\alpha_{i j}\left(M_{Z}\right)=b_{i j ; 00} \log \left(\frac{\Lambda}{M_{Z}}\right),
$$

the differential running of the coupling constants is unaffected by threshold corrections (it is the same at all the energy scales), and there is no scale of breaking.

In the described model, gauge unification is at best numerically achieved, rather then explained, by either identifying the cutoff scale with the "observed" unification scale $3 \times 10^{16} \mathrm{GeV}$, or by introducing threshold corrections at the cutoff scale, as shown in Fig. 1]

\subsection{Rototranslational orbifold: a $T^{2} / Z_{2}^{\prime}$ model with non-local gauge symmetry breaking}

We can consider the same $S U(5)$ theory on a different $T^{2} / Z_{2}^{\prime}$ orbifold, with $Z_{2}^{\prime}$ generated by $g^{\prime}$ with geometric action

$$
g^{\prime}: z_{1} \rightarrow z_{1}+\pi R_{1}, \quad g^{\prime}: z_{2} \rightarrow-z_{2},
$$

and gauge embedding $\gamma_{g^{\prime}}=\operatorname{Diag}(-1,-1,-1,1,1)$. Due to the translational action along $z_{1}, g^{\prime}$ acts freely, and the resulting orbifold has no singularities. Consider now the Kaluza-Klein expansion of a scalar field in the fundamental representation of 
$S U(5)$, given in Eq. (2). The $g^{\prime}$ action on the KK modes is such that the invariant states are

$$
\begin{array}{ll}
\Phi_{m_{1} m_{2}}^{D}+(-1)^{m_{1}} \Phi_{m_{1},-m_{2}}^{D} & \text { doublet, } \\
\Phi_{m_{1} m_{2}}^{T}-(-1)^{m_{1}} \Phi_{m_{1},-m_{2}}^{T} & \text { triplet. }
\end{array}
$$

The KK towers of surviving states are then given in Fig. 2. for $m_{2} \neq 0$ they are identical, i.e. for each mass level with $m_{2} \neq 0$ there is a complete multiplet of $S U(5)$. If $m_{2}=0$ the doublet $\Phi^{D}$ has KK tower $M^{2}=m_{1}^{2} / R_{1}^{2}$ with even $m_{1}$ 's, the triplet $\Phi^{T}$, instead, has tower $M^{2}=m_{1}^{2} / R_{1}^{2}$ with odd $m_{1}$ 's. One can check that given any bulk field in a multiplet of $S U(5)$ the KK expansion shows similar features. In detail, there are complete multiplets of $S U(5)$ for each KK level with $m_{2} \neq 0$, incomplete multiplets for $m_{2}=0$ levels. Moreover, in the $m_{2}=0$ case, the combination of the field content of any $m_{1}=$ even level with any $m_{1}=$ odd level gives again a complete multiplet of the $S U(5)$ group.

Given this, we can argue about the differential running of the coupling constants. The states with $m_{2} \neq 0$ are irrelevant, since they fill complete multiplets of $S U(5)$, i.e. $b_{i j ; m_{1} m_{2}}=0$ for $m_{2} \neq 0$. About the states with $m_{2}=0$, we have that a submultiplet with even $m_{1}$ and one with odd $m_{1}$ contribute with opposite coefficient, since they combine into a complete $S U(5)$ multiplet, whose contribution to the differential running is zero. We have then $b_{i j ; 2 m_{1} 0}=-b_{i j ; 2 m_{1}+10}$. Moreover, the absence of fixed points ensures the absence of extra localized matter so that the only contribution to $b_{i j ; 00}$ comes from the expansion of six-dimensional fields. This implies that the relation $b_{i j ; 2 m_{1} 0}=-b_{i j ; 2 m_{1}+10}$ holds also for $m_{1}=0$. Then Eq. (4) reduces to

$$
\alpha_{i j}=b_{i j ; 00} \log \frac{R_{1}^{-1}}{M_{Z}}+b_{i j ; 00} f\left[\frac{\Lambda}{R_{1}^{-1}}\right]
$$

with

$$
f[N]=\left(\log N+2 \sum_{n=1}^{N}(-1)^{n} \log \frac{N}{n}\right) .
$$

The function $|f[x]|$ is well defined for $x \rightarrow \infty$ and converges in that limit to $\log (2 / \pi)$. In other terms, the differential running due to the massless modes is completely canceled by the threshold corrections arising at the compactification scale $1 / R_{1}$, that can be identified with the unification scale. Thus, we conclude that in the described model the gauge symmetry breaking has a breaking (unification) scale $M_{G U T}=1 / R_{1}$.

Unfortunately, the described model cannot be relevant in model building, since the orbifold action on $z_{2}$ is just a translation, and this clashes with the requirement

\footnotetext{
d Notice that, from a string perspective, there is a $g^{\prime}$ twisted sector, but all the states have mass larger then $M_{0} \sim m_{s}^{2} R_{1}$, where $m_{s}$ is the string scale. Thus, these states are all heavier than the string scale (cutoff) provided that $R_{1}^{-1}<m_{s}$.
} 
of a four-dimensional chiral spectrum. Such a problem can be avoided by adding an extra orbifold operator, as discussed in the following section.

\section{3. $A T^{2} / Z_{2} \times Z_{2}^{\prime}$ model with non-local gauge symmetry breaking}

In order to have a chiral $4 \mathrm{~d}$ spectrum and non-local gauge symmetry breaking, we can combine the $Z_{2}$ projection described in Sect. 2.1 with the $Z_{2}^{\prime}$ projection described in Sect. 2.2. We have then a model with two orbifold projections, $g$ and $g^{\prime}$, acting as

$$
\begin{array}{ll}
g: z_{1} \rightarrow-z_{1}, & g: z_{2} \rightarrow-z_{2}+\delta, \\
g^{\prime}: z_{1} \rightarrow z_{1}+\pi R_{1}, & g^{\prime}: z_{2} \rightarrow-z_{2} .
\end{array}
$$

The requirement that $g$ and $g^{\prime}$ commute implies that $\delta=0$ or $\delta=\pi R_{2}$. In both cases the action of $g$ has fixed points in the internal space; since we want a nonlocal gauge symmetry breaking we define $\gamma_{g}$ to be the identity matrix. In this way $g$ does not break the unified gauge symmetry and, moreover, it projects away all the continuous Wilson lines. In order to have a gauge symmetry breaking we define, $\gamma_{g}^{\prime}=\operatorname{Diag}(-1,-1,-1,1,1)$, as done in Sect. 2.2.

The orbifold group contains the operators $g$ and $g^{\prime}$, the first with local geometric action and not breaking the gauge symmetry, the second with non-local action and breaking the gauge symmetry. It also contains the mixed operator $g \cdot g^{\prime}$, breaking the gauge symmetry. It has local action if $\delta=0$, non-local action if $\delta=\pi R$. In the latter case, the action is non-local since it is a translation along $z_{2}$ (and the resulting geometry is precisely the projective space described in 17 ). This implies that only in the second case the gauge symmetry breaking is completely non-locally realized. From this, we immediately conclude that in the second case the differential running of the coupling constants stops at a certain scale 17 . Still, it is interesting to check this via a direct computation, in order to obtain a precise relation between the symmetry breaking scale and the compactification scales.

The $\delta=0$ case: localized gauge symmetry breaking

In the $\delta=0$ case the KK expansion of a multiplet in the fundamental representation of $S U(5)$ is given by the invariant combinations

$$
\begin{aligned}
& \left(\Phi_{m_{1}, m_{2}}^{D}+\Phi_{-m_{1},-m_{2}}^{D}\right)+(-1)^{m_{1}}\left(\Phi_{m_{1},-m_{2}}^{D}+\Phi_{-m_{1}, m_{2}}^{D}\right), \\
& \left(\Phi_{m_{1}, m_{2}}^{T}+\Phi_{-m_{1},-m_{2}}^{T}\right)-(-1)^{m_{1}}\left(\Phi_{m_{1},-m_{2}}^{T}+\Phi_{-m_{1}, m_{2}}^{T}\right) .
\end{aligned}
$$

This means that the towers for the doublet $\Phi^{D}$ and the triplet $\Phi^{T}$ coincide for $m_{1}, m_{2} \neq 0$, and so these states are irrelevant for what concerns the differential running of the couplings. For $m_{1} \geq 0, m_{2}=0$, instead, the two towers are identical but shifted: even positive $m_{1}$ states in the doublet, odd positive $m_{1}$ states in the triplet. This is very similar to the situation studied in Sect. 2.2, but with the crucial difference that in that case $m_{1} \in \mathbb{Z}$, here the KK tower of invariant states has instead $m_{1} \in \mathbb{N}$. Due to this, the running of the coupling constants receive threshold 

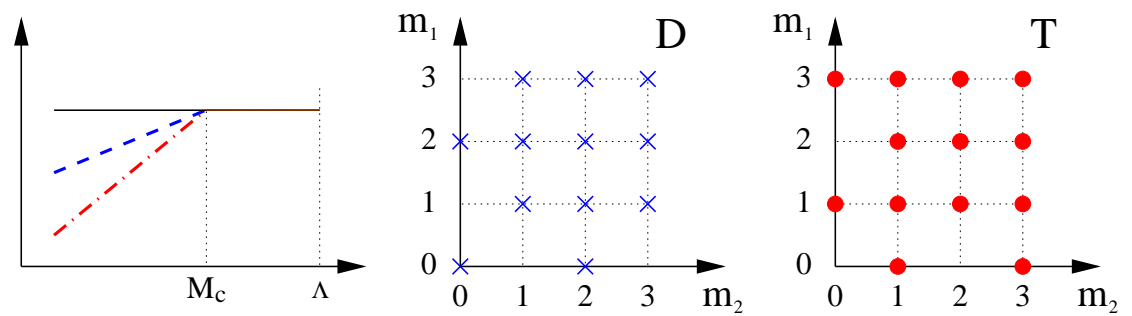

Fig. 3. Kaluza-Klein expansion of a fundamental representation of $S U(5)$ in the $T^{2} / Z_{2}$ orbifold. The figure in the middle shows the KK tower of the doublet (D), a blue cross in a point with coordinates $m_{1}, m_{2}$ indicates the presence in the tower of a single state with $K K$ mass $m^{2}=$ $m_{1}^{2} / R_{1}^{2}+m_{1}^{2} / R_{2}^{2}$. The figure on the right shows the tower for the triplet $(T)$. A similar expansion holds for any $S U(5)$ multiplets, this implies that the running of the difference of the inverse coupling constants is as given in the picture on the left, i.e. it precisely stops, due to the KK contributions, at the compactification scale $M_{C}=1 / \sqrt{R_{1} R_{2}}$. In the model the breaking scale is then reproduced and explained provided $M_{C}=M_{G U T}=3 \times 10^{16} \mathrm{GeV}$.

corrections, but they cancel only half of the differential running due to the zero modes (for a more direct geometric understanding of this result sec 20 ). Moreover, the remaining states with $m_{1}=0, m_{2}>0$, are present only in the doublet tower, making things even worse. Such a feature is due to the fact that, if $\delta=0$, the breaking due to $g \cdot g^{\prime}$ is localized not on isolated points in the internal space, but on the whole line $z_{2}=0, z_{1} \in S^{1}$. About the expansion of multiplets other then the fundamental, and the presence of localized extra matter, we can apply the same observation of previous Sections, and conclude that the differential running of the couplings will receive threshold corrections at the compactification scale, but that, generically, they are able to only partially stop the differential running due to the zero modese, and it is impossible to identify a breaking scale $M_{G U T}$ such that Eq. (4) reduces to Eq. (5).

The $\delta=\pi R_{2}$ case: non-local gauge symmetry breaking

If $\delta=\pi R_{2}$, then the invariant combinations are

$$
\begin{aligned}
& \left(\Phi_{m_{1}, m_{2}}^{D}+(-1)^{m_{2}} \Phi_{-m_{1},-m_{2}}^{D}\right)+(-1)^{m_{1}}\left(\Phi_{m_{1},-m_{2}}^{D}+(-1)^{m_{2}} \Phi_{-m_{1}, m_{2}}^{D}\right) \\
& \left(\Phi_{m_{1}, m_{2}}^{T}+(-1)^{m_{2}} \Phi_{-m_{1},-m_{2}}^{T}\right)-(-1)^{m_{1}}\left(\Phi_{m_{1},-m_{2}}^{T}+(-1)^{m_{2}} \Phi_{-m_{1}, m_{2}}^{T}\right)
\end{aligned}
$$

for the doublet $\Phi^{D}$ and the triplet $\Phi^{T}$ respectively. As shown in Fig. 3, for $m_{1}, m_{2} \neq 0$ we have no difference between the towers. For $m_{1}=0$ the doublet tower contains only even positive $m_{2}$ states, while the triplet tower contains only odd positive $m_{2}$ states, and similarly for $m_{2}=0$ : the doublet tower contains only even $m_{1}$ 's and the triplet only odd $m_{1}$ 's. An identical expansion is present for any bulk multiplet. Combining this with the absence of extra localized matter not filling

e Clearly, given a very specific choice of the localized matter, it is in principle possible to stop the differential running of the couplings, at least in the case $R_{2} \lesssim 1 / \Lambda$. 
$S U(5)$ multiplet: $f$, we conclude that the coefficient $b_{i j ; m_{1} m_{2}}$ in Eq. (44) are zero if both $m_{1}$ and $m_{2}$ are non-zero. If $m_{1}$ is zero $b_{i j ; 02 m_{2}}=-b_{i j ; 02 m_{2}+1}$. Similarly, if $m_{2}$ is zero, $b_{i j ; 2 m_{1} 0}=-b_{i j ; 2 m_{1}+10}$. Then Eq. (4) can be rewritten as

$$
\alpha_{i j}=b_{i j ; 00} \log \frac{\sqrt{R_{1}^{-1} R_{2}^{-1}}}{M_{Z}}+\frac{1}{2} b_{i j ; 00} f\left[\frac{\Lambda}{R_{1}^{-1}}\right]+\frac{1}{2} b_{i j ; 00} f\left[\frac{\Lambda}{R_{2}^{-1}}\right],
$$

where the function $f$ has been defined in Eq. (11). Given the properties of $f$ we deduce the existence of a unification (breaking) scale $M_{G U T}=\sqrt{R_{1}^{-1} R_{2}^{-1}}$.

\subsubsection{Generalization}

The explicit extension of the described computation to a generic orbifold is unfortunately not available. Still, from the purely geometric arguments given above, we can argue that in any model where the gauge symmetry breaking is non-local, i.e. all the symmetry breaking orbifold operators act as rototranslations, the differential running of the coupling constants stops at a scale set by the compactification moduli. In particular, in presence of $q$ orbifold operators responsible for the gauge symmetry breaking, non-locality can be invoked provided that each of them acts as a translation along some internal direction. The scale of breaking is then given by the volume $V$ of the submanifold spanned by such directions: $M_{G U T}=V^{-1 / q}$.

\subsubsection{Non-local breaking vs. discrete/continuous Wilson lines}

We remark that the mechanism is peculiarly different from a discrete Wilson line breaking. Consider, as an example, a $T^{2} / Z_{2} \times Z_{2}^{\prime}$ orbifold with orbifold operators

$$
\begin{array}{ll}
g: z_{1} \rightarrow-z_{1}, & g: z_{2} \rightarrow-z_{2} ; \\
g^{\prime}: z_{1} \rightarrow z_{1}+\pi R_{1}, & g^{\prime}: z_{2} \rightarrow z_{2} .
\end{array}
$$

The operator $g$ generates the orbifold $T^{2} / Z_{2}$, with four fixed points $\left(z_{1}, z_{2}\right)=(0,0)$, $\left(\pi R_{1}, 0\right),\left(0, \pi R_{2}\right),\left(\pi R_{1}, \pi R_{2}\right)$, where the breaking due to $g$ is localized. The operator $g^{\prime}$ introduces a discrete Wilson line along $z_{1}$. In other words, the fundamental space of $T^{2} / Z_{2} \times Z_{2}^{\prime}$ is the same as the one of $T^{2} / Z_{2}$ (with half volume) but the four singularities are now not equivalent: there are two fixed points $\left(z_{1}, z_{2}\right)=(0,0)$, $\left(0, \pi R_{2}\right)$ where the gauge symmetry breaking due to $g$ is localized, and two fixed points $\left(z_{1}, z_{2}\right)=\left(\pi R_{1}^{\prime}, 0\right),\left(\pi R_{1}^{\prime}, \pi R_{2}\right)$ where the breaking due to $g \cdot g^{\prime}$ is localized (we define $R_{1}^{\prime}=R_{1} / 2$ ). This immediately clarifies that in such an orbifold the breaking is always localized in points in the internal space: if $g$ breaks the gauge symmetry then such a breaking is localized in the first two fixed points, if instead $g \cdot g^{\prime}$ is responsible for the breaking, then it is localized in the second fixed points.

${ }^{\mathrm{f}}$ Indeed, all the singularities of the orbifold are due to orbifold rotations respecting the $S U(5)$ symmetry, and so the localized extra matter must fill complete $S U(5)$ multiplets. 
If neither $g$ nor $g \cdot g^{\prime}$ break the gauge symmetry, then no gauge symmetry breaking at all is present.

Given this, we conclude that a discrete Wilson line does not realize, in general, a non-local gauge symmetry breaking, and we cannot expect the presence of a scale of breaking.

This does not mean that any discrete Wilson line induces a breaking without a scale: in some very specific constructions such a feature can be achievedg. As a non-trivial example of this, in the last section we show an $S^{1} / Z_{2} \times Z_{2}^{\prime}$ orbifold with a rank reducing orbifold operator $g$ and a discrete Wilson line $g^{\prime}$ in which the breaking due to $g^{\prime}$ has a scale of breaking, precisely given by the compactification radius.

About the possibility that the described non-local gauge symmetry breaking may be completely reabsorbed into continuous Wilson lines, we remark that this is not the case in a semirealistic model with $\mathcal{N}=1$ SUSY in $4 \mathrm{~d}$. Indeed, the SUSY condition implies that for each internal direction $z^{m}$ there is an orbifold operator $g_{z^{m}}$ acting as a rotation on it. Since $g_{z^{m}}$ respects the SM gauge group, there are Wilson lines $A^{m}$ only outside the SM generators, i.e. outside the Cartan subalgebra of $S U(5)$. This implies that there can be continuous Wilson lines, but their gauge symmetry breaking always induce a rank reduction.

\section{String models with non-local gauge symmetry breaking}

We pass to the study of backgrounds with six internal dimensions, relevant in string model building: $T_{1}^{2} \times T_{2}^{2} \times T_{3}^{2} / Z_{M} \times Z_{N}^{\prime}$ orbifolds. The orbifold groups are generated by $g$ and $g^{\prime}$, and are such that both in heterotic and in Type I string case the gauge symmetry breaking due to $g^{\prime}$ is non-local. Indeed, the details of the breaking in these cases are exactly as in the field theory examples studied in previous section. This is due to the fact that, in heterotic and Type I string, the gauge degrees of freedom propagate in the whole $10 \mathrm{~d}$ spacetime. We comment in a separate subsection the gauge symmetry breaking that occurs, instead, in the case of Type II string with Dp-branes $(p<9)$. In all the cases the resulting four dimensional models have $\mathcal{N}=1$ SUSY. This is the minimal choice with the described features, since nonlocality and $\mathcal{N}=1$ SUSY cannot be simultaneously obtained in $T_{1}^{2} \times T_{2}^{2} \times T_{3}^{2} / Z_{M}$ models.

We parameterize each $T_{i}^{2}$ torus with a complex variable $z_{i}$, with periodicities $z_{i} \sim z_{i}+R_{i}, z_{i} \sim z_{i}+\tau_{i} R_{i}$. We allow non trivial complex structures $\tau_{i}$, previously set to $i R_{1} / R_{2}$ (rectangular torus). The orbifold group $Z_{M}$ is generated by $g$, a pure rotation acting on each complex variable as $g: z_{i} \rightarrow e^{2 \pi v_{i} / M_{2}} z_{i}, v_{i} \in \mathbb{Z}$. The orbifold group $Z_{N}^{\prime}$ is generated by $g^{\prime}$ acting as $g^{\prime}: z_{i} \rightarrow e^{2 \pi v_{i}^{\prime} / N} z_{i}+\delta_{i} / N, v_{i}^{\prime} \in \mathbb{Z}$, $\delta_{i}=a_{i}+b_{i} \tau_{i}, a_{i}, b_{i} \in \mathbb{Z}$. We fix $v_{1}^{\prime}=0, \delta_{i} \neq 0$ so that $g^{\prime}$ is a pure translation

${ }^{\mathrm{g}}$ This does not change our conclusion about the qualitative difference between discrete Wilson lines and non-local symmetry breaking: only in the latter the existence of a breaking scale is a generic feature. 
along $z_{1}$. Since $g$ has local action and $g^{\prime}$ non local action, the gauge symmetry breaking due to $g$ is localized, the one due to $g^{\prime}$ is instead non-local. Since the orbifold group contains also the mixed orbifold operators $g^{n} \cdot g^{\prime m}$, the breaking due to $g^{\prime}$ is really non-local only provided that all such operators (with $m \neq 0$ ) act as rototranslations in the internal space. This imposes strong constraints on $v, v^{\prime}$ and $\delta$, to be combined with those on $v$ and $v^{\prime}$ ensuring $\mathcal{N}=1$ SUSY in $4 \mathrm{~d}$, and with those on $\delta$ ensuring that the orbifold group is Abelian. Examples of orbifolds fulfilling all these conditions are

\section{- $\mathrm{T}^{6} / \mathrm{Z}_{2} \times \mathrm{Z}_{2}^{\prime}$}

In this case $v=(1,1,0), v^{\prime}=(0,1,1), \delta=(1,1,0)$ औ. There are two gauge symmetry breaking orbifold operators, $g^{\prime}$ and $g g^{\prime}$, both acting as rototranslations. The symmetry breaking scale is $M_{G U T}=\left(R_{1} R_{2}\right)^{-1 / 2}$. The described geometry is shown in Fig. 4.

\section{- $\mathrm{T}^{6} / \mathrm{Z}_{4} \times \mathrm{Z}_{2}^{\prime}$}

In this case $v=(1,1,-2), v^{\prime}=(0,1,1), \delta=\left(1+\tau_{1}, 1+\tau_{2}, 1 / 2\right)$ with $\tau_{1}=\tau_{2}=i$. There are four gauge symmetry breaking orbifold operators, $g^{n} g^{\prime}$ for $n=0, \ldots, 3$, all acting as rototranslations. The symmetry breaking scale is $M_{G U T}=\left(R_{1} R_{2} R_{3}\right)^{-1 / 3}$.

\section{- $\mathrm{T}^{6} / \mathrm{Z}_{3} \times \mathrm{Z}_{3}^{\prime}$}

In this case $v=(1,1,0), v^{\prime}=(0,1,1), \delta_{i}=1+\tau_{i}, \tau_{i}=e^{2 \pi i / 3}$. All the operators $g^{n} g^{\prime m}$ with $m \neq 0$ break the GUT symmetry and act as rototranslations. The symmetry breaking scale is $M_{G U T}=\left(R_{1} R_{2} R_{3}\right)^{-1 / 3}$.

\subsection{Open strings on freely acting orbifolds: internal vs external orbifold action}

In type II string theory the gauge degrees of freedom propagate on Dp-branes, hypersurfaces of $p+1$-dimensionality. In case $p<9$, then, the orbifold action can be internal to a single stack of D-branes, i.e. it can map it to itself, but it can also be external, i.e. it can map a stack of D-branes into a different one, localized at a different point in the internal space. In the first case the physics is as described in the field theory examples given in previous section. In the second case there is a rank reduction of the gauge group: if an orbifold action is external to a stack of D-branes, then it maps it into a different stack, identifying the corresponding gauge groups. As an example, consider a stack $A$ of D-branes with $U(5)_{A}$ gauge group, a stack $B$ of D-branes with $U(5)_{B}$ gauge group and an orbifold operator $g$ mapping $A$ in $B$. Whatever the action of $g$ is, the unbroken gauge symmetry is $U(5)$, combination of

\footnotetext{
${ }^{\mathrm{h}}$ It is easy to check that the most general translation vector $\delta_{i}=\left(a_{i}+b_{i} \tau_{i}\right) / 2$ fulfills all the conditions provided that $\delta_{1} \neq 0$ and at least one of the other two entries is non-zero.

i Notice that the two stacks must be identical, otherwise the orbifold action identifying $A$ with $B$ cannot be a symmetry of the system.
} 


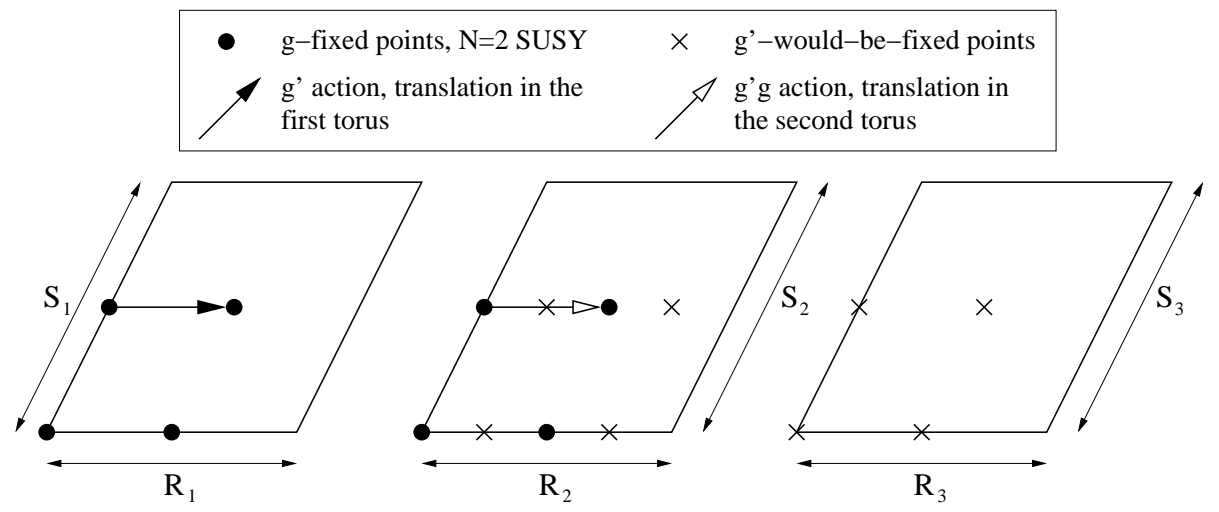

Fig. 4. Internal geometry of the $Z_{2} \times Z_{2}^{\prime}$ model. The action of $g$ is not free, the dots show its fixed points in the first and second torus; since $g$ has no action in the third torus each point $z_{3} \in \mathbb{C}$ is a (4d) fixed point preserving $\mathcal{N}=2 S U S Y$. The arrows show the action of $g^{\prime}\left(g g^{\prime}\right)$ in the first (second) torus: a pure translation. The crosses show the would-be-fixed points of $g^{\prime}$.

$U(5)_{A}$ with $U(5)_{B}$. Notice that, if $g$ is a rotation, we can continuously move the $A$ and $B$ stacks till they coincide, in a fixed point of $g$, a gauge enhancement occurs and the gauge symmetry breaking due to $g$ is a standard rank-preserving symmetrybreaking of the enhanced $U(10)$ gauge group (notice that the surviving gauge group is also an enhancement of $U(5))$. In this sense, in the purely rotational case, the picture is completely dual to the $p=9$ case, with the displacement between $A$ and $B$ stacks replaced by a continuous rank-reducing Wilson line. If instead the action of $g$ is a translation, then the distance between $A$ and $B$ is fixed and no gauge enhancement is possiblej.

From the description above we conclude that an orbifold model with only internal orbifold actions can be completely described as in the field theory examples given in previous sections. If instead the orbifold actions are completely external, then the gauge symmetry breaking always includes a rank reduction, and a breaking of the kind $S U(5) \rightarrow S U(3) \times S U(2) \times U(1)$ is never possible. The case with both internal and external orbifold actions, instead, is more intriguing, and we show the possibilities that it offers in the following subsection, with an explicit example.

Notice that the description of the gauge symmetry breaking in presence of internal or external translational orbifold actions (also called parallel and perpendicular actions respectively) is very close to the SUSY breaking one (as an example, see ${ }^{11}$ ).

${ }^{j}$ In other words, in the dual picture, the corresponding continuous rank-reducing Wilson line is projected out of the spectrum by $g$, but $g$ itself can be seen as a sort of discrete rank-reducing Wilson line. 

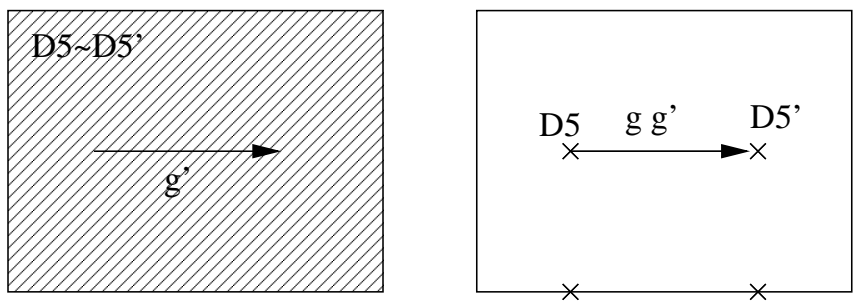

Fig. 5. The background described in Fig. 4 with two stacks of 5 D5-branes filling the first torus and located in two would-be-fixed points of $g^{\prime}$ in the second torus (the third torus is not shown, the D5-branes all sit in $g^{\prime}$-fixed points).

\subsubsection{A $Z_{2} \times Z_{2}^{\prime}$ model with mixed internal and external orbifold action: localized breaking with a breaking scale}

The combination of internal and external orbifold actions is peculiar, since in this case a discrete Wilson lines is naturally combined with a rank-reducing orbifold projection. As an example, we consider the $Z_{2} \times Z_{2}^{\prime}$ geometry of Fig. 4 and embed two stacks of 5 D5-branes, filling the first torus and localized into two different would-be-fixed points of $g^{\prime}$ in the second torus, as shown in Fig. [5. The gauge group, in absence of any orbifold projection, is $U(5)_{D 5} \times U(5)_{D 5^{\prime}}$. The action of $g^{\prime}$ is free and internal, since it maps each stack of D-branes into itself. We can embed it into the gauge degrees of freedom such that $g^{\prime}: U(5) \rightarrow U(3) \times U(2)$ both for D5 and D $5{ }^{\prime}$ -branes. The action of $g$ is instead non-free and external, since it maps one stack of D-branes into the other in the second torus, so that $g: U(5)_{D 5} \times U(5)_{D 5^{\prime}} \rightarrow U(5)_{g}$. Similarly, the action of $g g^{\prime}$ is also external, and induces an identification between the two $U(5)$ 's, but the breaking is $g g^{\prime}: U(5)_{D 5} \times U(5)_{D 5^{\prime}} \rightarrow U(5)_{g^{\prime}}$, such that $U(5)_{g} \cap U(5)_{g^{\prime}}=U(3) \times U(2)$, so that the ending unbroken group is then just the latter group.

It is interesting to reproduce these features in a simplified field theory model, with a single extra dimension parameterized by $x \sim x+2 \pi R$ and compactified on $S^{1} / Z_{2} \times Z_{2}^{1 \mathrm{k}}$. The action of the $Z_{2}$ operator is $g: x \rightarrow-x$, with fixed points 0 and $\pi R$, while the action of the $Z_{2}^{\prime}$ operator is $g^{\prime}: x \rightarrow x+\pi R$. Thus, the mixed operator $g g^{\prime}$ has fixed points $\pi R / 2$ and $3 \pi R / 2$ (see Fig. 6) and $g^{\prime}$ can be seen as a discrete Wilson line. The bulk gauge symmetry is $S U(5)_{1} \times S U(5)_{2}, g$ acts on the gauge group such that $g: S U(5)_{1} \leftrightarrow S U(5)_{2}$. More precisely, defining $T_{i}^{a b}$ a generator of $S U(5)$. 1 the identification is $g: T_{1}^{a b} \leftrightarrow T_{2}^{a b}$, and the surviving gauge group in 0 and $\pi R$ is a diagonal $S U(5)$ generated by $T_{1}^{a b}+T_{2}^{a b}$. We embed then $Z_{2}^{\prime}$ action as $g^{\prime}: T_{1}^{a b} \rightarrow \delta_{c}^{a} T_{2}^{c d} \delta_{d}^{b}$ with $\delta$ a diagonal matrix: $\delta=\operatorname{Diag}[-1,-1,-1,1,1]$.

${ }^{\mathrm{k}}$ It should be possible to obtain such a field theory model, with $S U(5)$ groups rather then $U(5)$ groups, from the described open string model, by shrinking the second and third torus, and the vertical direction of the first torus, and neglecting the corresponding KK states.

${ }^{1}$ We take $a, b=1,2 \ldots, 5$, and define $T^{a a}$ as the 5 Cartan generators of $U(5)$, from which we exclude the "diagonal" generator $\sum_{a} T^{a a}$. 


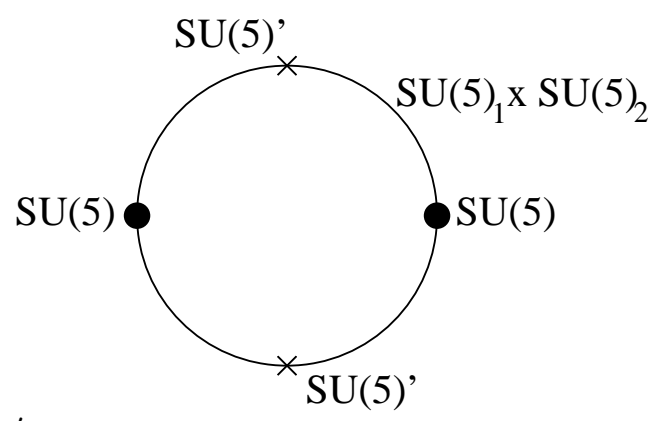

Fig. 6. An $S^{1} / Z_{2} \times Z_{2}^{\prime}$ field theory model. The bulk symmetry is $S U(5)_{1} \times S U(5)_{2}$, broken to $S U(5)$ and to $S U(5)^{\prime}$ in the $g$ and $g g^{\prime}$ fixed points (dots and crosses respectively). The surviving gauge group is just the $S M$ gauge group: $S U(5) \cap S U(5)^{\prime}=S U(3) \times S U(2) \times U(1)$.

In this way the surviving gauge group in $\pi R / 2$ and $3 \pi R / 2$ is the one left invariant by the operator $g g^{\prime}$ and it is a different $S U(5)^{\prime}$, such that the intersection of the two gauge groups is just $S U(3) \times S U(2) \times U(1)$, the Standard Model (SM) gauge group. The breaking is local, since the $S U(5)$ symmetry preserved in 0 is generically broken in $\pi R / 2$, but the differential running of the coupling constants is generated only by the bulk degrees of freedom, and it stops precisely at the scale $M_{G U T}=R^{-1}$. There is no fixed-point-contribution to the differential running since only full multiplets of $S U(5)\left(S U(5)^{\prime}\right)$ can be localized there, and the SM gauge group is embedded exactly in the same way in $S U(5)$ and in $S U(5)^{\prime}$. The last point is crucial: a multiplet of $S U(5)$ behaves as a multiplet of $S U(5)^{\prime}$ under the action of the SM gauge group, and they both contribute universally to the running of the coupling constants. We expect the same argument to be valid for any localized contribution to the action. Thus, in this model there is a scale of breaking, given by the length of the unique compact dimension $M_{G U T}=R^{-1}$.

The described mechanism can be embedded, in principle, also in an heterotic string theory context, in presence of rank-reducing continuous Wilson lines. We can indeed have an orbifold model with $U(10)$ gauge symmetry in the bulk (the breaking $S O(32) \rightarrow U(10) \times \mathcal{G}_{\text {Hidden }}$ can be realized via discrete Wilson lines), and two orbifold operators $g$ and $g^{\prime}$. We take $g$ such that it breaks $U(10)$ to $U(5) \times U(5)$, and $g^{\prime}$, freely acting, such that each $U(5)$ is broken to $U(3) \times U(2)$. We can choose $g$ and $g^{\prime}$ such that any combination $g^{n} g^{\prime m}$ breaks $U(10)$ to some $U(5)^{\prime} \times U(5)^{\prime}$. In this way all the singularities in the orbifold respect some $U(5)$ symmetry, and the scale of breaking $U(5) \times U(5) \rightarrow U(3) \times U(2) \times U(3) \times U(2)$ is set by a single internal dimension $R_{g^{\prime}}$. By adding a continuous Wilson line we can then identify the two $U(5)$ factors, and have a model with a massless $U(3) \times U(2)$ gauge group, with coupling unification at $1 / R_{g^{\prime}}$, and an extra twin gauge group $U(3)^{\prime} \times U(2)^{\prime}$, broken by the continuous Wilson line. Notice also that such a model can arise only in $S O(32)$ heterotic string, where examples of orbifold models with $U(10)$ (or $S O(20)$ ) gauge groups are present 21 . 


\section{References}

1. H. Georgi, H. R. Quinn and S. Weinberg, Phys. Rev. Lett. 33 (1974) 451;

S. Dimopoulos, S. Raby and F. Wilczek, Phys. Rev. D 24 (1981) 1681.

2. J. R. Ellis, S. Kelley and D. V. Nanopoulos, Phys. Lett. B 249, 441 (1990);

U. Amaldi, W. de Boer and H. Fürstenau, Phys. Lett. B 260, 447 (1991);

P. Langacker and M. X. Luo, Phys. Rev. D 44, 817 (1991).

3. H. Georgi and S. L. Glashow, Phys. Rev. Lett. 32, 438 (1974);

H. Georgi, in Proceedings, Coral Gables 1975, Theories and Experiments In High Energy Physics, New York (1975);

J. C. Pati and A. Salam, Phys. Rev. D 8 (1973) 1240; Phys. Rev. D 10 (1974) 275.

4. Y. Kawamura, Prog. Theor. Phys. 105 (2001) 999 arXiv:hep-ph/0012125;

G. Altarelli and F. Feruglio, Phys. Lett. B 511 (2001) 257 arXiv:hep-ph/0102301];

L. J. Hall and Y. Nomura, Phys. Rev. D 64, 055003 (2001) arXiv:hep-ph/0103125.

5. A. Hebecker and J. March-Russell, Nucl. Phys. B 613 (2001) 3 arXiv:hep-ph/0106166.

6. T. Asaka, W. Buchmüller and L. Covi, Phys. Lett. B 523 (2001) 199 arXiv:hep-ph/0108021;

L. J. Hall, Y. Nomura, T. Okui and D. R. Smith, Phys. Rev. D 65 (2002) 035008 arXiv:hep-ph/0108071.

7. T. Kobayashi, S. Raby and R. J. Zhang, Nucl. Phys. B $\mathbf{7 0 4}$ (2005) 3 arXiv:hep-ph/0409098; Phys. Lett. B 593 (2004) 262 arXiv:hep-ph/0403065;

S. Förste, H. P. Nilles, P. K. S. Vaudrevange and A. Wingerter, arXiv:hep-th/0406208, W. Buchmuller, K. Hamaguchi, O. Lebedev and M. Ratz, Nucl. Phys. B 712 (2005) 139 arXiv:hep-ph/0412318; Phys. Rev. Lett. 96 (2006) 121602 arXiv:hep-ph/0511035; arXiv:hep-ph/0512326, arXiv:hep-th/0606187.

8. Y. Hosotani, Phys. Lett. B 126 (1983) 309; Annals Phys. 190 (1989) 233;

E. Witten, Nucl. Phys. B 258 (1985) 75.

9. J. Scherk and J. H. Schwarz, Nucl. Phys. B 153 (1979) 61;

R. Rohm, Nucl. Phys. B 237, 553 (1984);

C. Kounnas and M. Porrati, Nucl. Phys. B 310, 355 (1988);

S. Ferrara, C. Kounnas, M. Porrati and F. Zwirner, Nucl. Phys. B 318, 75 (1989);

C. Kounnas and B. Rostand, Nucl. Phys. B 341 (1990) 641;

I. Antoniadis, E. Dudas and A. Sagnotti, Phys. Lett. B 464, 38 (1999) arXiv:hep-th/9908023;

10. E. Kiritsis and C. Kounnas, Nucl. Phys. B 503, 117 (1997) arXiv:hep-th/9703059;

C. A. Scrucca and M. Serone, JHEP 0110, 017 (2001) arXiv:hep-th/0107159;

C. A. Scrucca, M. Serone and M. Trapletti, Nucl. Phys. B 635, 33 (2002) arXiv:hep-th/0203190;

P. Anastasopoulos, A. B. Hammou and N. Irges, Phys. Lett. B 581 (2004) 248 arXiv:hep-th/0310277;

C. Angelantonj, M. Cardella and N. Irges, Nucl. Phys. B 725, 115 (2005) arXiv:hep-th/0503179.

11. I. Antoniadis, G. D’Appollonio, E. Dudas and A. Sagnotti, Nucl. Phys. B 565, 123 (2000) arXiv:hep-th/9907184;

C. Angelantonj, R. Blumenhagen and M. R. Gaberdiel, Nucl. Phys. B 589 (2000) 545 arXiv:hep-th/0006033.

12. A. Pomarol and M. Quiros, Phys. Lett. B 438, 255 (1998) arXiv:hep-ph/9806263;

I. Antoniadis, S. Dimopoulos, A. Pomarol and M. Quiros, Nucl. Phys. B 544, 503 (1999) arXiv:hep-ph/9810410;

A. Delgado, A. Pomarol and M. Quiros, Phys. Rev. D 60, 095008 (1999) 
arXiv:hep-ph/9812489;

R. Barbieri, L. J. Hall and Y. Nomura, Phys. Rev. D 63, 105007 (2001) arXiv:hep-ph/0011311.

13. L. J. Hall and Y. Nomura, Phys. Rev. D 65 (2002) 125012 arXiv:hep-ph/0111068.

14. V. S. Kaplunovsky, Phys. Rev. Lett. 55 (1985) 1036.

15. L. J. Dixon, V. Kaplunovsky and J. Louis, Nucl. Phys. B 355 (1991) 649;

I. Antoniadis, K. S. Narain and T. R. Taylor, Phys. Lett. B 267 (1991) 37;

L. E. Ibanez, D. Lüst and G. G. Ross, Phys. Lett. B 272 (1991) 251 arXiv:hep-th/9109053;

P. Mayr, H. P. Nilles and S. Stieberger, Phys. Lett. B 317, 53 (1993) arXiv:hep-th/9307171;

H. P. Nilles and S. Stieberger, Nucl. Phys. B 499, 3 (1997) arXiv:hep-th/9702110;

Phys. Lett. B 367, 126 (1996) arXiv:hep-th/9510009;

M. K. Gaillard and R. L. Xiu, Phys. Lett. B 296 (1992) 71 arXiv:hep-ph/9206206;

G. Lopes Cardoso, D. Lüst and T. Mohaupt, Nucl. Phys. B 450 (1995) 115 arXiv:hep-th/9412209;

D. Ghilencea and G. G. Ross, Nucl. Phys. B 569 (2000) 391 arXiv:hep-ph/9908369;

Nucl. Phys. B 606 (2001) 101 arXiv:hep-ph/0102306.

16. A. E. Faraggi, Phys. Lett. B 302 (1993) 202 arXiv:hep-ph/9301268;

K. R. Dienes and A. E. Faraggi, Phys. Rev. Lett. 75 (1995) 2646 arXiv:hep-th/9505018; N Nucl. Phys. B 457 (1995) 409 arXiv:hep-th/9505046];

K. R. Dienes, A. E. Faraggi and J. March-Russell, Nucl. Phys. B 467 (1996) 44 arXiv:hep-th/9510223;

G. B. Cleaver, A. E. Faraggi and D. V. Nanopoulos, Phys. Lett. B 455 (1999) 135 arXiv:hep-ph/9811427.

17. A. Hebecker, JHEP 0401, 047 (2004) arXiv:hep-ph/0309313.

18. A. Hebecker and M. Trapletti, Nucl. Phys. B 713 (2005) 173 arXiv:hep-th/0411131].

19. M. Trapletti, Nucl. Phys. B 730 (2005) 179 arXiv:hep-th/0507151.

20. A. Hebecker and A. Westphal, Annals Phys. 305 (2003) 119 arXiv:hep-ph/0212175.

21. K. S. Choi, S. Groot Nibbelink and M. Trapletti, JHEP 0412 (2004) 063 arXiv:hep-th/0410232. 